\title{
A Nonequilibrium Rate Formula for Collective Motions of Complex Molecular Systems
}

\author{
Tomohiro Yanao*, Wang Sang Koon ${ }^{\dagger}$ and Jerrold E. Marsden ${ }^{\dagger}$ \\ ${ }^{*}$ Department of Applied Mechanics and Aerospace Engineering, Waseda University, Tokyo 169-8555, Japan \\ ${ }^{\dagger}$ Control and Dynamical Systems, MC 107-81, California Institute of Technology, Pasadena, CA 91125, USA
}

\begin{abstract}
We propose a compact reaction rate formula that accounts for a non-equilibrium distribution of residence times of complex molecules, based on a detailed study of the coarse-grained phase space of a reaction coordinate. We take the structural transition dynamics of a six-atom Morse cluster between two isomers as a prototype of multi-dimensional molecular reactions. Residence time distribution of one of the isomers shows an exponential decay, while that of the other isomer deviates largely from the exponential form and has multiple peaks. Our rate formula explains such equilibrium and non-equilibrium distributions of residence times in terms of the rates of diffusions of energy and the phase of the oscillations of the reaction coordinate. Rapid diffusions of energy and the phase generally give rise to the exponential decay of residence time distribution, while slow diffusions give rise to a non-exponential decay with multiple peaks. We finally make a conjecture about a general relationship between the rates of the diffusions and the symmetry of molecular mass distributions.
\end{abstract}

Keywords: reaction rate, reaction coordinate, non-RRKM, dimension reduction, hyperspherical coordinates, diffusion, cluster PACS: 82.20.-w, 05.45.-a, 36.40.-c

\section{INTRODUCTION}

Understanding the mechanisms and rates of conformational transitions of molecular systems is a significant challenge in molecular and biomolecular sciences. The standard reaction rate theories, such as the transition state theory and the RRKM theory [1], postulate that the internal energy of a molecule is redistributed rapidly among intramolecular degrees of freedom to achieve an equilibrium state, and as a result, the distribution of residence time (lifetime) of a reactant is in the exponential form. However, recently, molecular reactions that occur in non-equilibrium regimes start to attract significant interest in various fields such as bond-selective control of reactions [2], femtochemistry [3], and single-mode excitation of peptides [4]. The purpose of the present study is to shed light on such non-equilibrium rate processes of complex molecular systems from the viewpoint of coarse-grained phase-space dynamics and energy transfer.

The model we study here is a six-atom Morse cluster called $\mathrm{M}_{6}$, which serves as a prototype of complex molecular systems. This cluster has two geometrically distinct isomers called OCT (octahedron) and CTBP (capped trigonal bipyramid), which are depicted in the insets of Fig. 1. The potential energy minimum of OCT is $V=-12.49 \varepsilon$ and that of CTBP is $V=-12.13 \varepsilon$, where $\varepsilon$ is the unit of energy that determines the depth of the pairwise Morse potential. We adopt the constant-energy (microcanonical) molecular dynamics simulations at total energy $E=-11.0 \varepsilon$ for this system throughout this study.
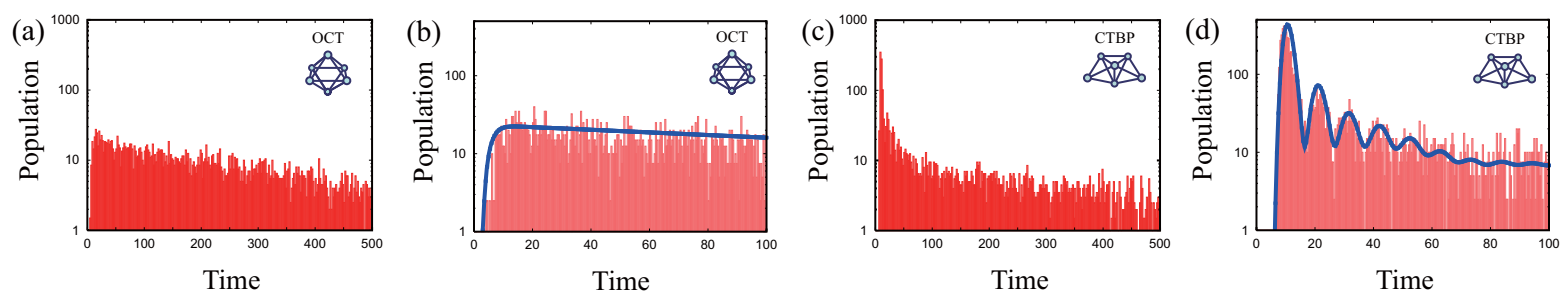

FIGURE 1. Distribution of residence times in OCT ((a) and (b)) and CTBP ((c) and (d)) of the six-atom Morse cluster. (b) and (d) are magnifications of (a) and (c) respectively for the short time range. The bar graphs represent numerical results, while the thick solid curves in (b) and (d) are plots obtained from the rate formula, Eq. (1). 
Interestingly, the residence time (lifetime) distributions in the two isomers of the $\mathrm{M}_{6}$ cluster have quite different characteristics as shown in Fig. 1(a)(b) for OCT and Fig. 1(c)(d) for CTBP. To obtain these figures, we first sent in an ensemble of classical trajectories in the phase space of OCT and CTBP respectively at time $t=0$, and measured the number of trajectories (per unit time) that transit into the other isomer at respective times. As can be seen from Fig. 1(a) and (b), the residence time distribution in OCT shows an exponential decay. On the other hand, the residence time distribution in CTBP shown in Fig. 1 (c) and (d) deviates largely from the exponential form. Moreover, there exist multiple peaks in the short-time range of the distribution in CTBP as can be seen from Fig. 1(d). Recently, this type of non-statistical residence time distributions have been computed and scrutinized in terms of the geometry of phase space in a number of studies $[5,6,7]$. While these studies tend to focus on the numerical computations of rates for rather low dimensional systems (typically with 3 degrees of freedom), the present study tries to clarify the physical mechanisms behind the exponential and non-exponential distributions of a relatively high dimensional system (with 12 degrees of freedom) from the viewpoint of a coarse-grained phase space of a reaction coordinate.

\section{REACTION COORDINATE AND COARSE-GRAINED PHASE SPACE}

We construct here a coarse-grained phase space of a reaction coordinate for the $\mathrm{M}_{6}$ cluster based on the method presented in Refs. $[9,10]$. This method built on the framework of the hyperspherical coordinates [8], which parametrize the $3 n-6$ internal degrees of freedom of an $n$-atom system by 3 gyration radii, $\mathbf{a} \equiv\left(a_{1}, a_{2}, a_{3}\right)^{T}$, and the $3 n-9$ hyperangles. For molecular reactions, in which a system changes its overall mass distribution in a significant way, one can generally construct a good reaction coordinate by using a combination of the three gyration radii. Specifically for the $\mathrm{M}_{6}$ cluster, we constructed a reaction coordinate through the linear transformation of the three gyration radii as $\mathbf{a}^{\prime}=\mathrm{Ga}$, where $\mathbf{a}^{\prime} \equiv\left(a_{1}^{\prime}, a_{2}^{\prime}, a_{3}^{\prime}\right)^{T}$ are the transformed gyration radii and $\mathrm{G}$ is a $3 \times 3$ orthogonal matrix determined by the principal component analysis of the dynamics of the three gyration radii (see Ref. [10] for the explicit expression of $\mathrm{G})$. After this transformation, $a_{1}^{\prime}$ plays the role of a reaction coordinate that dominate the structural transitions between the two isomers, while $a_{2}^{\prime}$ and $a_{3}^{\prime}$ can be regarded as "bath modes".

Figure 2(a) shows the vector field averaged at respective points in the phase space of the reaction coordinate $a_{1}^{\prime}$, where the vertical axis $p_{1}^{\prime}$ is the conjugate momentum of $a_{1}^{\prime}$. Here, the average is taken over an ensemble of long-time trajectories that undergo structural transitions between OCT and CTBP many times. Therefore, the averaged vector field represents the statistical (equilibrium) tendency of the behaviors of the trajectories projected onto the $a_{1}^{\prime}-p_{1}^{\prime}$ space. Fig. 2(a) clearly shows that the $a_{1}^{\prime}-p_{1}^{\prime}$ space possesses the essential properties of the phase space of a reaction coordinate, having two oscillatory regions separated by the saddle region at around $\left(a_{1}^{\prime}, p_{1}^{\prime}\right)=(2.5,0)$. The oscillatory region in the left of Fig. 2(a) corresponds to OCT, while the oscillatory region in the right corresponds to CTBP. Thus, the structural transition dynamics of the $\mathrm{M}_{6}$ cluster is characterized by the transitions of trajectories in the $a_{1}^{\prime}-p_{1}^{\prime}$ space between the left and the right oscillatory regions of Fig. 2(a).

It is important to note that the vector field in Fig. 2(a) is an averaged one, and in the actual dynamics of the cluster, instantaneous vector at each point in the $a_{1}^{\prime}-p_{1}^{\prime}$ space is indeed time-dependent and fluctuates around the average vector
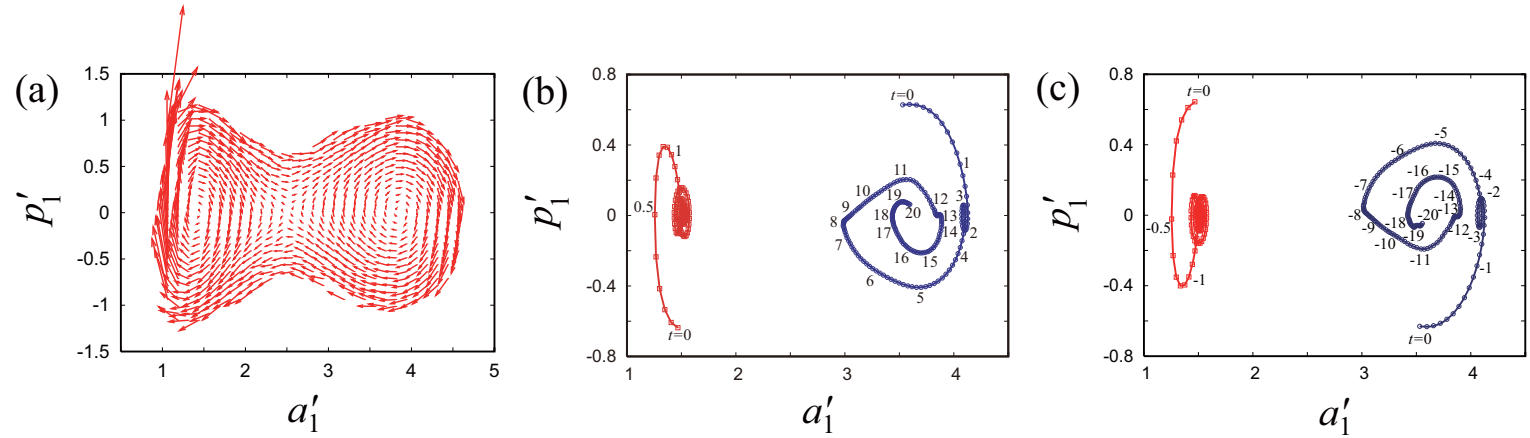

FIGURE 2. (a) Average vector field in the phase space of the reaction coordinate, the $a_{1}^{\prime}-p_{1}^{\prime}$ space, where the left region corresponds to OCT and the right region corresponds to CTBP. (b) Averaged paths that the trajectories entered into OCT (left curve) and CTBP (right curve) follow. (c) Averaged paths that the reactive trajectories from OCT (left curve) and CTBP (right curve) follow. 
of Fig. 2(a), depending on the coupling with all other internal degrees of freedom, $a_{2}^{\prime}, a_{3}^{\prime}$ and the 9 hyperangles. Thus, these fluctuations of the instantaneous vector field induce diffusions of nearby trajectories in the $a_{1}^{\prime}-p_{1}^{\prime}$ space. By noting that the trajectories have the common tendency to rotate clockwise in each isomer region of Fig. 2(a), we can classify the diffusions of the trajectories into radial and angular directions in each isomer region. The diffusion in the radial direction represents the diffusion (dissipation and acquisition) of energy in the reaction coordinate, while the diffusion in angular direction is the diffusion of the phase of the oscillation of the reaction coordinate.

Theoretically, we expect that the fluctuations of the vector field in the OCT region in the $a_{1}^{\prime}$ - $p_{1}^{\prime}$ space are much more significant than the fluctuations in the CTBP region. This is because, as was shown in Refs. [9, 10], a very strong impulsive force called the internal centrifugal force arises frequently, in addition to the static potential force, in the dynamics of gyration radii around the molecular conformations with symmetric mass distributions like OCT. On the other hand, since the mass distribution of CTBP is not symmetric, the internal centrifugal force is generally not very strong in CTBP. Therefore, the vector field in the CTBP region in the $a_{1}^{\prime}-p_{1}^{\prime}$ space does not fluctuate largely from the average.

The above theoretical expectations on the magnitudes of the fluctuations of the vector field can be readily confirmed as follows. Fig. 2(b) shows averaged paths in the $a_{1}^{\prime}-p_{1}^{\prime}$ space, where the average is taken over ensembles of trajectories that have just entered into the respective isomer regions and started to rotate from the points on the line $a_{1}^{\prime}=$ $1.5\left(p_{1}^{\prime}<0\right)$ for OCT and on the line $a_{1}^{\prime}=3.5\left(p_{1}^{\prime}>0\right)$ for CTBP. The numbers labeled along the averaged paths represent the elapsed time since the trajectories started to rotate. In the same way, Fig. 2(c) shows the paths averaged over an ensemble of reactive trajectories that get out of the isomer region and react right after they cross the line $a_{1}^{\prime}=1.5\left(p_{1}^{\prime}>0\right)$ for OCT and $a_{1}^{\prime}=3.5\left(p_{1}^{\prime}<0\right)$ for CTBP.

As can be seen from Fig. 2(b) and (c), the averaged paths for OCT quickly spiral into and out of the center around $\left(a_{1}^{\prime}, p_{1}^{\prime}\right)=(1.5,0)$. This fact clearly shows that the energy and the phase of the oscillation of the reaction coordinate diffuse fast due to the large fluctuations of the vector field in OCT. On the other hand, the averaged paths for CTBP spiral into and out of the central region around $\left(a_{1}^{\prime}, p_{1}^{\prime}\right)=(3.5,0)$ slowly compared to OCT. This fact shows that the reaction coordinate exchanges energy with other degrees of freedom slowly and the diffusion of the phase is also slow due to the small fluctuations of the vector field in CTBP. In the next section, we clarify the relationship between the rates of the diffusions and the residence time distributions shown in Fig. 1.

\section{A NON-EQUILIBRIUM RATE FORMULA}

We present here a compact reaction rate formula that accounts for the residence time distributions in Fig. 1 based on the observations in the previous section. Suppose we have $N_{0}$ trajectories that enter into an isomer region in the $a_{1}^{\prime}-p_{1}^{\prime}$ space and start to rotate from the points on the line $a_{1}^{\prime}=1.5\left(p_{1}^{\prime}<0\right)$ for OCT and on the line $a_{1}^{\prime}=3.5\left(p_{1}^{\prime}>0\right)$ for CTBP at time $t=0$. Let $\omega$ be the typical (average) angular frequency of the rotations of these trajectories in the isomer region. We also assume that the rotation angles of the trajectories, i.e., the phases of the oscillations of the reaction coordinate, diffuse with a Gaussian distribution whose mean squared displacement evolves as $\sigma(t)^{2}=2 D t$, where $D$ is the diffusion coefficient. Moreover, we let $\Phi_{i}$ be the probability for the trajectories to react in the $i$-th rotation. Then the overall reactive flux at time $t$, which is essentially the residence time distribution, is written as the summation of the time-dependent Gaussian distributions;

$$
F(t)=\sum_{k=1}^{\infty} \frac{\omega N_{0} \Phi_{k} \prod_{i=1}^{k-1}\left(1-\Phi_{i}\right)}{\sqrt{2 \pi \sigma(t)^{2}}} \exp \left[-\frac{\left\{2 \pi\left(k-\frac{1}{2}\right)-\omega\left(t-t_{0}\right)\right\}^{2}}{2 \sigma(t)^{2}}\right] .
$$

Figs. 1(b) and (d) compare this formula Eq. (1) (solid curves) with the numerical results of the residence time distributions (bar graphs) for the two isomers. The values of the parameters in Eq. (1) adopted here are summarized in Table 1. The total number of sample trajectories are $N_{0}=7292$ for OCT and $N_{0}=7275$ for CTBP. The angular frequency of the reaction coordinate is estimated to be $\omega=3.5$ for OCT and $\omega=0.6$ for CTBP from the oscillations

TABLE 1. Parameters for the rate formula Eq. (1) to reproduce the solid curves in Fig. 1(b) and (d).

\begin{tabular}{cccccccccccccc}
\hline Isomer & $N_{0}$ & $\omega$ & $D$ & $t_{0}$ & $\Phi_{1}$ & $\Phi_{2}$ & $\Phi_{3}$ & $\Phi_{4}$ & $\Phi_{5}$ & $\Phi_{6}$ & $\Phi_{7}$ & $\cdots$ & $\Phi_{100}$ \\
\hline OCT & 7292 & 3.5 & 5.0 & 5.5 & 0.01 & 0.01 & 0.01 & 0.01 & 0.01 & 0.01 & 0.01 & $\cdots$ & 0.01 \\
CTBP & 7275 & 0.6 & 0.045 & 5.5 & 0.3 & 0.1 & 0.06 & 0.05 & 0.04 & 0.03 & 0.025 & $\cdots$ & 0.025 \\
\hline
\end{tabular}


of the average paths in Figs. 2(b) and (c). The diffusion coefficient for the diffusion of the phase is set to $D=5.0$ for OCT and to $D=0.045$ for CTBP, indicating that the phase diffuses much faster in OCT than in CTBP due to the large fluctuations of the vector field in OCT as discussed in the previous section. The parameter $t_{0}$ accounts for the average time for the trajectories to pass through the transition region and is necessary here since this time is included in the numerical results of the residence time distributions in Fig. 1. The summation in Eq. (1) is taken from $k=1$ to $k=100$ for both OCT and CTBP. This means that we take into consideration the first 100 oscillations of the reaction coordinate and ignore all other oscillations after $k=101$.

The reaction probabilities for the respective numbers of oscillations of the reaction coordinate are $\Phi_{1}=\cdots=\Phi_{100}=$ 0.01 for OCT. That is, all $\Phi_{i}$ are the same irrespective of the number of the oscillations. This reflects the fact that the reaction coordinate loses energy quickly and the system is equilibrated immediately after the system has entered into the phase space of OCT. This equality of $\Phi_{i}$ is crucial for the exponential decay of the curve Eq. (1) as shown in Fig. 1(b). On the other hand, for CTBP, reaction probability $\Phi_{i}$ depends on the number of oscillations $i$ and decreases as $i$ increases from $i=1$ to 6 , and $\Phi_{i}$ is constant after $i=7$. The higher reaction probability for the smaller number of oscillations is due to the fact that the reaction coordinate tends to have higher energy immediately after the system has entered into CTBP and takes about the first 6 oscillations to lose energy and become equilibrated. As we see from Figs. 1(b) and (d), the rate formula Eq. (1) reproduces both the exponential (OCT) and the non-expnential (CTBP) distributions of residence times fairly well.

\section{CONCLUDING REMARKS}

We have clarified the mechanisms for the emergence of a non-statistical distribution of residence times of clusters in terms of the coarse-grained phase space of a reaction coordinate. Based on the notions of diffusions of energy and the phase of the oscillation of the reaction coordinate, we have proposed a simple rate formula that accounts for both the exponential and non-exponential distributions of residence times. If the energy and the phase of the reaction coordinate diffuse fast as in OCT, residence time distribution takes exponential form. On the other hand, if these diffusions are slow as in CTBP, residence time distribution becomes non-exponential with multiple peaks.

The results of the present study could be generalized as follows. In symmetric isomers like OCT, where two or three of the gyration radii are close to each other, the diffusions of energy and the phase of a reaction coordinate is generally fast due to large fluctuations of the internal centrifugal force. Therefore, we expect that the residence time distributions of isomers with symmetrical mass distributions tend to be in exponential form and obey the RRKM theory. On the other hand, in isomers with asymmetric mass distributions like CTBP, the internal centrifugal force may not be significant as compared to symmetric isomers, and as a result, the diffusion of energy and the phase of a reaction coordinate may not be fast. Therefore, we expect that the residence time distributions of isomers with asymmetric mass distributions tend to deviate from exponential form, where non-equilibrium effects play important roles. It is interesting to test this conjecture for different complex molecular systems such as fullerenes and biomolecules.

\section{ACKNOWLEDGMENTS}

This work has been partially supported by JSPS Grant-in-Aid for Research Activity Start-Up No. 21840051 as well as NSF Grants: NSF-DMS-0505711 and JEM.CMMI-1-NSF.DYNSYST No. 0926001.

\section{REFERENCES}

1. J. I. Steinfeld, J. S. Francisco, and W. L. Hase, Chemical Kinetics and Dynamics, Prentice-Hall, Englewood Cliffs, NJ, 1989.

2. D. R. Killelea, V. L. Campbell, N. S. Shuman, and A. L. Utz, Science, 319, 790 (2008).

3. E. W. -G. Diau, J. L. Herek, Z. H. Kim, and A. H. Zewail, Science 279, 847 (1998).

4. B. C. Dian, A. Longarte, and T. S. Zwier, Science, 296, 2369 (2002).

5. H. Waalkens, A. Burbanks, and S. Wiggins, Phys. Rev. Lett. 95, 084301 (2005).

6. F. Gabern, W. S. Koon, J. E. Marsden, and S. D. Ross, Physica D, 211, 391 (2005).

7. G. S. Ezra, H. Waalkens, and S. Wiggins, J. Chem. Phys. 130, 164118 (2009).

8. X. Chapuisat and A. Nauts, Phys. Rev. A 44, 1328 (1991).

9. T. Yanao, W. S. Koon, J. E. Marsden, and I. G. Kevrekidis, J. Chem. Phys. 126, 124102 (2007).

10. T. Yanao, W. S. Koon, and J. E. Marsden, J. Chem. Phys. 130, 144111 (2009). 